\title{
Osteoblast-derived Laminin-332 is a novel negative regulator of osteoclastogenesis in bone microenvironments
}

\author{
Norihisa Uehara', Akiko Kukita², Yukari Kyumoto-Nakamura', Takayoshi Yamaza ${ }^{1}$, Hisataka Yasuda ${ }^{3}$ and Toshio Kukita ${ }^{1}$
}

Laminin-332 (Lm-332), a major basement membrane protein, has been shown to provide a niche for some stem cells. Here, we found that Lm-332 was expressed in osteoblasts, and is implicated in the regulation of osteoclast differentiation. Immunofluorescence analysis of laminin- $\beta 3$, a unique component of $\mathrm{Lm}-332$, indicated specific expression of laminin- $\beta 3$ in osteoblast-like cells localized on bone surface. RT-PCR analysis confirmed that $\alpha 3, \beta 3$, and $\gamma 2$ chains of Lm-332 were all expressed in primary osteoblasts prepared from mouse calvaria. Lm-332 markedly inhibited osteoclastogenesis induced by receptor activator of nuclear factor kappa B (NF-KB) ligand (RANKL) when bone marrow-derived macrophages (BMMs) were cultured on Lm-332-coated plates. Lm-332 also blocked RANKL-induced activation of mitogen-activated protein kinases (MAPKs) (ERK, JNK, and p38) and expression of NFATc1, c-Fos, and c-Jun. Lm-332 suppressed osteoclast differentiation while retaining macrophage phenotypes, including nonspecific esterase activity and gene expression of lysozyme and EGF-like module-containing mucin-like hormone receptor-like 1 (Emr1). Furthermore, the treatment of primary osteoblasts with osteoclastogenic factors dramatically suppressed expression of Lm-332. These findings suggest that Lm-332 produced by osteoblasts in bone tissues has a pivotal role in controlling normal bone remodeling through suppressing osteoclastogenesis.

Laboratory Investigation (2017) 97, 1235-1244; doi:10.1038/labinvest.2017.55; published online 5 June 2017

Maintenance of hematopoietic stem cells (HSCs) and differentiation of progenitor cells takes place in specialized bone marrow microenvironments known as 'niches'. ${ }^{1}$ Adhesive interaction of hematopoietic stem and progenitor cells (HSPCs) with extracellular matrix (ECM) ligands produced by non-hematopoietic stromal cells is involved in controlling homeostasis of the HSC population. ${ }^{2}$ HSPCs and precursors of all blood cell lineages express adhesion receptors that enable cell-cell and cell-ECM interactions, and activate downstream signaling pathways, which mediate various cellular functions. ${ }^{3}$

Laminins, major ECM proteins mainly located in the basement membrane, are heterotrimeric glycoproteins composed of three covalently linked chains, $\alpha, \beta$, and $\gamma$. Their unique cruciate structures contribute to the organization of the ECM especially in basement membrane. ${ }^{4}$ To date, 16 laminin isoforms have been identified; these are composed of various combinations of each chain $(\alpha 1-5, \beta 1-3$, and $\gamma 1-3) .^{5}$
Laminin isoforms bind to various cell surface receptors, which include integrins, syndecans, and dystroglycans. Binding of laminins to their receptors transduces adhesion signaling and regulates cellular functions. Most studies on the interaction between laminins and various hematopoietic cells including macrophages ${ }^{6}$ have been performed using Engelbreth-Holm-Swarm (EHS) tumor-derived laminin-111 (Lm-111). Other laminin family proteins, such as Lm-311, Lm-511, and Lm-521, have also been demonstrated to be expressed in human bone marrow microenvironments. ${ }^{7}$ Lm-511 and Lm-521 bind to human CD34+ hematopoietic progenitor cells, and affect mitogenic activity and migration of these cells through $\alpha 6 \beta 1$ integrin. ${ }^{7,8}$ In contrast, Lm-332, which consists of laminin $\alpha 3, \beta 3$, and $\gamma 2$ chains, is known to be an important component present in skin epidermal basement membrane. ${ }^{9}$ Lm-332 has recently been shown to comprise a component of the 'niche' for a number of tissuespecific stem cells. Lm-332 is abundant beneath the

${ }^{1}$ Department of Molecular Cell Biology and Oral Anatomy, Division of Oral Biological Sciences, Faculty of Dental Science, Kyushu University, Fukuoka, Japan; ${ }^{2}$ Department of Microbiology, Faculty of Medicine, Saga University, Saga, Japan and B3ioindustry Division, Oriental Yeast Company, Limited, Tokyo, Japan

Correspondence: Professor T Kukita, PhD, Department of Molecular Cell Biology and Oral Anatomy, Division of Oral Biological Sciences, Faculty of Dental Science, Kyushu University, 3-1-1 Maidashi, Higashi-ku, Fukuoka 812-8582, Japan.

E-mail: kukitat@dent.kyushu-u.ac.jp

Received 27 February 2017; revised 6 April 2017; accepted 6 April 2017 
interfollicular epidermis, where it maintains hair follicle stem cells through the action of integrin-linked kinase (ILK), which is a key regulator of the bulge ECM microenvironments. ${ }^{10}$ Lm-332 is also a component of the niche for human hepatic cancer stem cells ${ }^{11}$ and for tumor cells in B-cell chronic lymphocytic leukemia. ${ }^{12}$ Although the expression of Lm-332 has been observed in cultures of human mesenchymal stem cells ${ }^{13}$ and bone marrow stromal cells, ${ }^{14}$ it is unclear whether Lm-332 is expressed in bone tissues or bone marrow tissues in vivo.

Osteoclasts, multinuclear giant cells specialized in bone resorption, are derived from HSCs, and in normal osteoclastogenesis, differentiate from a common precursor of macrophages and osteoclasts. Their differentiation is basically supported by two essential cytokines, receptor activator of $\mathrm{NF}-\kappa \mathrm{B}$ ligand (RANKL), and macrophage colony-stimulating factor (M-CSF) ${ }^{15-17}$ produced by cells in the osteoblast lineage. ${ }^{18}$ Intracellular signaling after binding of RANKL to its receptor RANK has been extensively studied. In brief, TNF receptor-associated factor 6 (TRAF6) activates downstream signaling, including MAPK pathways, c-Jun-N-terminal kinase (JNK), and p38MAPK. Subsequently, this signaling activates transcription factors such as c-Fos and c-Jun, components of activator protein-1 (AP-1), ${ }^{19,20}$ and NFATc $1,{ }^{21}$ which induce the expression of genes required for osteoclastogenesis. In addition to RANKL signaling, cell adhesion signaling through ECM proteins contributes to fine regulation of osteoclast differentiation. ${ }^{22}$ Of the receptors for ECM proteins, integrins, composed of heterodimeric adhesion molecules with $\alpha$ and $\beta$ subunits, have been extensively studied. Integrin $\alpha \mathrm{v} \beta 3$ expressed in osteoclasts recognizes the Arg-Gly-Asp (RGD) consensus sequence of ECM proteins, such as vitronectin and osteopontin, ${ }^{22-24}$ which is thought to enable osteoclasts to bind bone surface and to perform bone resorption. Osteoclast precursors circulate throughout the vascular system. We have previously found that Lm-111, a component of the basement membrane of blood vessels, suppresses osteoclastogenesis. We have postulated that the differentiation of osteoclasts is negatively regulated by Lm-111 localized in the basement membrane of blood vessels present throughout the vascular system. ${ }^{25}$

Here we investigated the expression of Lm-332 in bone tissues, and we examined the role of Lm-332 in the regulation of osteoclast differentiation. We found that Lm-332 is expressed in osteoblasts, and has an important regulatory role in osteoclastogenesis. Our findings suggest the presence of a novel mechanism operating through Lm-332 that controls normal bone remodeling.

\section{MATERIALS AND METHODS}

\section{Animals}

C57BL/6 mice were obtained from Kyudo (Tosu, Japan). Animal care and experiments were conducted in accordance with guidelines and procedures approved by the Institutional
Animal Care and Use Committee of Kyushu University (protocol number: A27-179-0, A27-179-1).

\section{Immunohistochemical Analysis}

Femurs were excised from 5- to 6-week-old male C57BL/6 mice and immersed in $10 \%$ formalin neutral buffer solution for $24 \mathrm{~h}$ and demineralized in $10 \%(\mathrm{w} / \mathrm{v})$ ethylene diamine tetraacetic acid disodium salt (EDTA-2Na; Dojindo) at room temperature for a week. The samples were dehydrated with graded ethanol and embedded in paraffin. For immunohistochemistry, the tissue sections of femurs were deparaffinized, rehydrated, and soaked in HistoVT One antigen retrieval solution (Nacalai, Kyoto, Japan). After blocking with Blocking One Histo (Nacalai), the sections were incubated with rabbit polyclonal antibody to laminin $\beta 3$ (GeneTex, Irvine, CA, USA) at $4{ }^{\circ} \mathrm{C}$ overnight in a humidified chamber, followed by three washes with TBS-Tween (TBST). The sections were further incubated with goat anti-rabbit IgG antibody conjugated with Alexa Fluor 488 (Molecular Probes, Eugene, OR, USA) and counterstained with DAPI for nuclei. The sections were mounted with Fluoromount/Plus (Diagnostic Biosystems, Pleasanton, CA, USA). Images were acquired on a Carl Zeiss fluorescent microscope using the Apotome function and ZEN software (Carl Zeiss, Jena, Germany).

\section{Preparation and Culture of Osteoblasts}

Primary osteoblasts were prepared from calvariae of 3-5-dayold C57BL/6 mice by five sequential digestions using $0.1 \%$ collagenase I (Wako Pure Chemicals). The cells were grown for 21 days in $\alpha$-MEM containing 10\% FBS in the presence of $\beta$-glycerophosphate and ascorbic acid (Wako Pure Chemicals). Alkaline phosphatase (ALP) activity was examined using TRAP/ALP Staining kit (Wako Pure Chemicals) according to the protocol provided in the kit. For $1,25(\mathrm{OH})_{2} \mathrm{D}_{3}$ or PTH stimulation, primary osteoblasts were treated with $10 \mathrm{nM}$ of $1,25(\mathrm{OH})_{2} \mathrm{D}_{3}$ (Cayman, Ann Arbor, MI, USA) or $100 \mathrm{ng} / \mathrm{ml}$ of human PTH (1-31) (Bachem Bioscience, King of Prussia, PA, USA) as indicated in each experiment.

\section{Immunofluorescence Analysis}

Immunofluorescence analysis was performed as described previously. ${ }^{26}$ Calvarial osteoblasts prepared from 3- to 5-dayold C57BL/6 mice were grown for 21 days on $35 \mathrm{~mm}$ glass bottom dishes (Matsunami Glass, Osaka, Japan) in $\alpha$-MEM containing $10 \%$ FBS in the presence of $\beta$-glycerophosphate and ascorbic acid. After fixation with $10 \%$ formalin neutral buffer solution for $10 \mathrm{~min}$, the cells were washed with TBST and blocked with Blocking One Histo (Nacalai) for $10 \mathrm{~min}$. The cells were incubated with rabbit polyclonal antibody to laminin $\beta 3$ overnight at $4^{\circ} \mathrm{C}$. After washing with TBS, $0.1 \%$ Tween, cells were incubated with anti-rabbit IgG antibody conjugated with Alexa Fluor 488 (Molecular Probes) at room temperature for $1 \mathrm{~h}$. The cells were then counterstained with DAPI for $5 \mathrm{~min}$. Cells were imaged using BZ-8100 fluorescence microscope (Keyence). Non-immune 
rabbit IgG (DAKO, Carpinteria, CA, USA) was used as the negative control in place of a primary antibody.

\section{Cell Culture}

Murine osteoclast precursor cell line, RAW264 D clone (RAW-D $)^{27,28}$ was maintained in $\alpha$-minimal essential medium $(\alpha$-MEM) (Life Technologies, Carlsbad, CA, USA) containing $10 \%$ fetal bovine serum (FBS; Life Technologies) supplemented with $100 \mathrm{U} / \mathrm{ml}$ of penicillin and $100 \mathrm{mg} / \mathrm{ml}$ of streptomycin at $37^{\circ} \mathrm{C}$ in a $95 \%$ air $/ 5 \% \mathrm{CO}_{2}$ atmosphere. The human recombinant Laminin-5 (Lm-332) was obtained from Oriental Yeast (Tokyo, Japan). The human Lm-511-E8 was obtained from Nippi (Tokyo, Japan). Culture plates were coated with each ECM protein overnight at $4{ }^{\circ} \mathrm{C}$ and washed twice with PBS before use.

\section{Preparation of BMMs and In Vitro Osteoclastogenesis}

The femurs and tibiae were excised from 5-6-week-old male C57BL/6 mice. Bone marrow cells were cultured for $24 \mathrm{~h}$ in $\alpha$-MEM containing $10 \%$ FBS in the presence of M-CSF $(20 \mathrm{ng} / \mathrm{ml})$. Non-adherent cells were collected and seeded in a $100 \mathrm{~mm}$ dish with $\alpha$-MEM containing $10 \%$ FBS and $20 \mathrm{ng} / \mathrm{ml}$ M-CSF (Peprotech, London, UK). After $72 \mathrm{~h}$, adherent cells were used as BMMs. For osteoclastogenesis, BMMs were further cultured in the presence of $20 \mathrm{ng} / \mathrm{ml} \mathrm{M}$-CSF and

Table 1 List of primers used for real-time PCR analysis

\begin{tabular}{|c|c|c|}
\hline $\begin{array}{l}\text { Gene } \\
\text { name }\end{array}$ & Forward primer & Reverse primer \\
\hline Trap & CAGCCCTTACTACCGTTTGC & GTAGTCCTCCTTGGCTGCTG \\
\hline Ctsk & AGGCGGCTATATGACCACTG & TCTTCAGGGCTTTCTCGTTC \\
\hline ATP6VOd2 & CACGGAGATGAGGAAGAAGC & TGGGTGACACTTGGCTAGAA \\
\hline Dcstamp & GTATCGGCTCATCTCCTCCA & GGAACCCAAGTCTCAGACACA \\
\hline $\operatorname{ltg} 33$ & ACAGAGCGTGTCCCGTAATC & GTCTTCCATCCAGGGCAATA \\
\hline Calcr & TCTTGGTTGGAGAGGTGCTT & TCGGTTTCTTCTCCTCTGGA \\
\hline Nfatcl & GGGAAGAAGATGGTGCTGTC & TTGCGGAAAGGTGGTATCTC \\
\hline$c-f o s$ & GGCAGAAGGGGCAAAGTAG & GTTGATCTGTCTCCGCTTGG \\
\hline Rank & CCAGGAGAGGCATTATGAGC & СTTCATTCCAGGTGTCCAAG \\
\hline Emr1 & TTGTACGTGCAACTCAGGACT & GATCCCAGAGTGTTGATGCAA \\
\hline Lyz & ATGGAATGGCTGGCTACTATGG & ACCAGTATCGGCTATTGATCTGA \\
\hline Runx2 & AGGGACTATGGCGTCAAACA & GGCTCACGTCGCTCATCTT \\
\hline Ocn & TAGCAGACACCATGAGGACCCT & TGGACATGAAGGCTITGTCAGA \\
\hline Rankl & CAGCATCGCTCTGTTCCTGTA & CTGCGTTTCATGGAGTCTCA \\
\hline Opg & ACCCAGAAACTGGTCATCAGC & CTGCAATACACACACTCATCACT \\
\hline Lama3 & CTGTGACTACTGCAATTCTGAGG & CAAGGTGAGGTTGACTTGATTGT \\
\hline Lamb3 & GGCTGCCTCGAAATTACAACA & ACCCTCCATGTCTTGCCAAAG \\
\hline Lamc2 & CAGACACGGGAGATTGCTACT & CCACGTTCCCCAAAGGGAT \\
\hline Actb & CCCTGAAGTACCCCATTGAA & GGGGTGTTGAAGGTCTCAAA \\
\hline$B 2 m$ & CCTGGTCTTTCTGGTGCTTG & CCGTTCTTCAGCATTTGGAT \\
\hline
\end{tabular}

$50 \mathrm{ng} / \mathrm{ml}$ RANKL (Peprotech) for 4-5 days, and RAW-D cells were cultured in the presence of $50 \mathrm{ng} / \mathrm{ml}$ RANKL for 3 days. BMMs were plated onto 96-well plates and cultured in the presence of M-CSF and RANKL for up to 5 days. The cells were fixed and stained using the Acid Phosphatase, Leukocyte (TRAP) Kit (Sigma, Saint-Louis, MO, USA). The numbers of TRAP-positive multinuclear osteoclasts (with more than three nuclei) per well were counted.

\section{Actin Ring Staining}

BMMs were cultured in 96-well plates in the presence of M-CSF and RANKL for 4-5 days. The cells were then fixed with $10 \%$ formalin neutral buffer solution (WAKO, Osaka, Japan) and stained with fluorescein-labeled phalloidin (Life Technologies) for F-actin and with 4',6-diamidino-2-phenylindole (DAPI; Dojindo Kumamoto, Japan) for nuclei. Images were captured using a BZ-8100 fluorescence microscope (Keyence, Osaka, Japan).

\section{Quantitative Real-Time PCR Analysis}

The mRNA expression was analyzed by quantitative real-time PCR with the Thermal Cycler Dice (TAKARA, Shiga, Japan). Total RNA was prepared using an RNeasy Mini kit (Qiagen, Valencia, CA, USA), and the cDNA was then reversetranscribed using a SuperScript III first-strand cDNA synthesis kit (Life Technologies). PCR amplification was performed in triplicate using a KAPA SYBR FAST qPCR kit (KAPA Biosystems, Boston, MA, USA). The PCR conditions were as follows: enzyme activation at $95^{\circ} \mathrm{C}$ for $30 \mathrm{~s}, 40$ cycles of $5 \mathrm{~s}$ at $95^{\circ} \mathrm{C}$ and $20 \mathrm{~s}$ at $60^{\circ} \mathrm{C}$. Expression was calculated using the comparative threshold cycle (CT) method. ${ }^{29}$ Primer sequences used in this study are shown in Table 1.

\section{Cell Proliferation Analysis}

Viable cells were identified using the PrestoBlue fluorescent dye (Life Technologies) according to the manufacturer's instructions. BMMs and RAW-D cells were plated at $1.5 \times 10^{4}$ and $5 \times 10^{3}$ cells/well, respectively, on 96-well plates coated with/without Lm-332. BMMs and RAW-D cells were cultured for 4 and 3 days, respectively, and PrestoBlue fluorescent dye was added to each well, followed by incubation for $1 \mathrm{~h}$ at $37^{\circ} \mathrm{C}$. The fluorescence was read $(\operatorname{Ex} 560 \mathrm{~nm} / \mathrm{Em} 590 \mathrm{~nm})$ on a plate reader (Infinite 200; Tecan, Switzerland).

\section{Western Blot Analysis}

Western blots were performed as described previously. ${ }^{30}$ Cell lysates were prepared by homogenization in cell lysis buffer (Cell Signaling Technology, Beverly, MA, USA) supplemented with protease inhibitor cocktail. Nuclear and cytoplasmic proteins were extracted by NE-PER nuclear and cytoplasmic extraction kit (Thermo Scientific, Rockford, IL, USA). The protein concentration of the supernatants was measured using a detergent-compatible protein assay kit (Bio-Rad). The following primary antibodies were used: anti-c-Jun, antiLamin A/C, anti-PARP-1, anti-Tubulin, anti-phospho-p44/42 
a
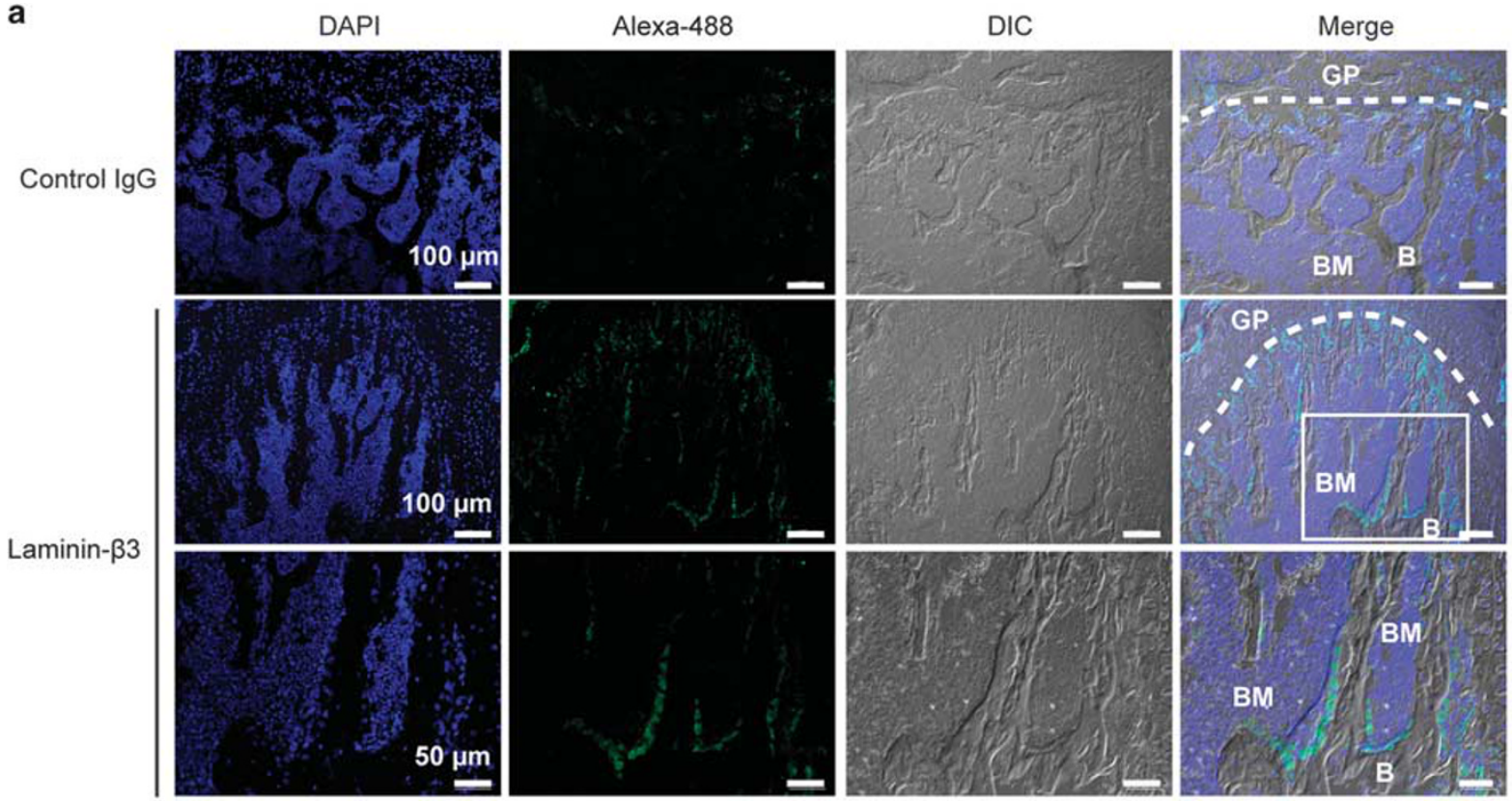

b
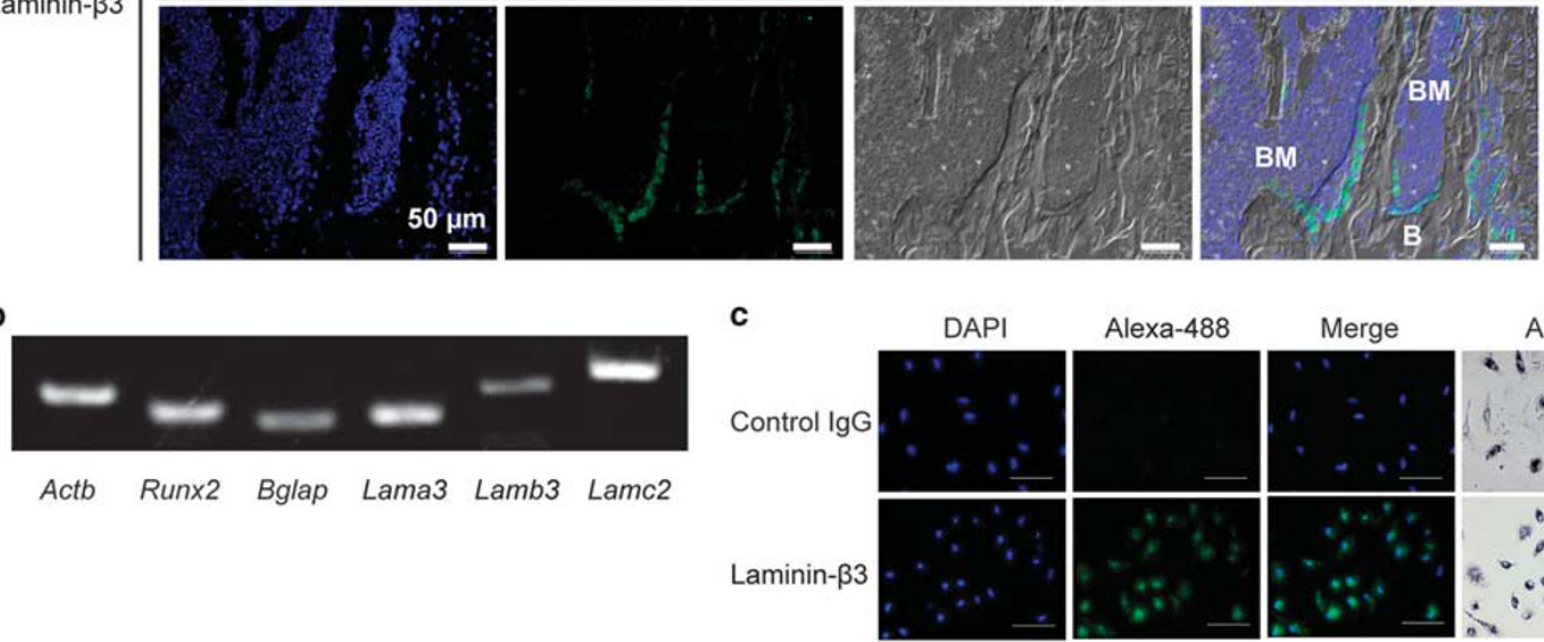

Runx2 Bglap Lama3 Lamb3 Lamc2
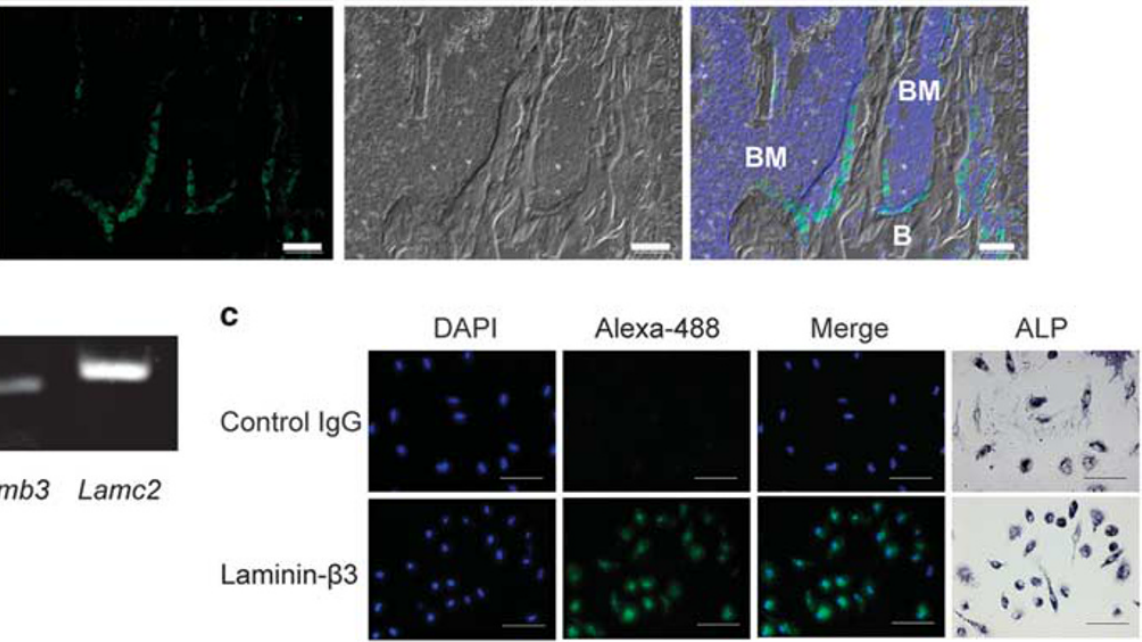

Figure 1 Expression of Lm-332 in murine osteoblasts. (a) Immunohistochemical analysis. Longitudinal sections of femur were prepared from 5-week-old male mice. The sections were stained with control non-immune lgG (upper panels) or anti-laminin $\beta 3$ chain antibody (lgG) (middle and lower panels) and detected with anti-rabbit lgG conjugated with Alexa Fluor 488 (green). Nuclei were stained with DAPI (blue). Data show the metaphyseal regions of distal femur. Higher magnification of rectangle region is shown in lower panels. Bars, $100 \mu \mathrm{m}$ (upper and middle panels); 50 (lower panels). B, bone; BM, bone marrow; GP, growth plate. Dashed lines mark growth plate. (b) Osteoblasts prepared from calvaria of newborn mice were cultured in 24-well plates in the presence of $2.5 \mathrm{mM} \beta$-glycerophosphate and $50 \mu \mathrm{g} / \mathrm{ml}$ ascorbic acid for 14 days to form mature osteoblasts. RT-PCR was used to amplify the indicated mRNA transcripts from mouse osteoblasts (14 days). The Runx2 and osteocalcin (Bglap) were used as a positive control for osteoblast maturation. $\beta$-actin (Actb) was amplified as a positive control. (c) The cells were fixed and stained for laminin- $\beta 3$ and ALP as described in the 'Materials and Methods' section.

MAPK (Thr202/Tyr204), anti-phospho-p38MAPK antibody (Thr180/Tyr182), anti-p38, anti-phospho-SAPK/JNK (Thr183/ Tyr185), and anti-SAPK/JNK, (Cell Signaling Technology); and anti-NFATc1, anti-c-Fos, anti-ERK1, and HRP-conjugated polyclonal anti-Actin antibodies (Santa Cruz Biotechnology, Santa Cruz, CA, USA).

\section{Statistical Analysis}

All discrete values are expressed as mean \pm standard deviation (s.d.) from at least three independent experiments. The data were analyzed by paired $t$-test for comparison between two groups or by one-way analysis of variance (ANOVA) followed by the Tukey's test for multiple comparisons. $P<0.05$ was considered as statistically significant.

\section{RESULTS}

\section{Expression of Lm-332 in Bone Tissues}

As Lm-332 is the only laminim isoform that contains the $\beta 3$ chain, we examined the localization of Lm-332 protein in bone tissues using an antibody specific for laminin $\beta 3$ chain. Laminin $\beta 3$ antibody selectively stained cuboidal osteoblastlike cells observed on surface of the bone in the metaphyseal area of the distal femur, but did not stain bone marrow (Figure 1a, lower panels). Control IgG failed to stain any component of the bone (Figure 1a, upper panels). These data suggest that Lm-332 was expressed in cells in osteoblast lineage in bone tissues. To confirm the expression of Lm-332 in osteoblasts, expression of laminin $\alpha 3, \beta 3$, and $\gamma 2$ chains in primary osteoblasts was examined by immunostaining and PCR analysis. Calvarial-derived primary osteoblasts were 
a

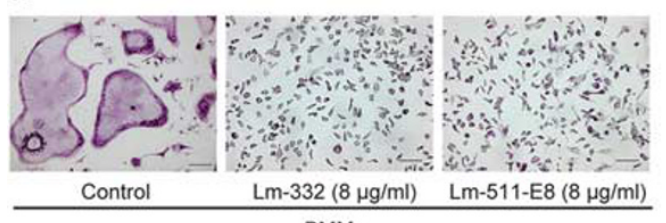

BMMs
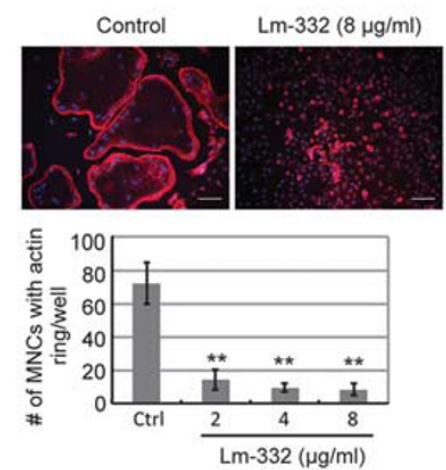

b

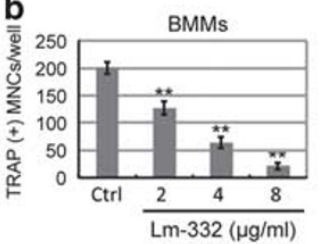

d

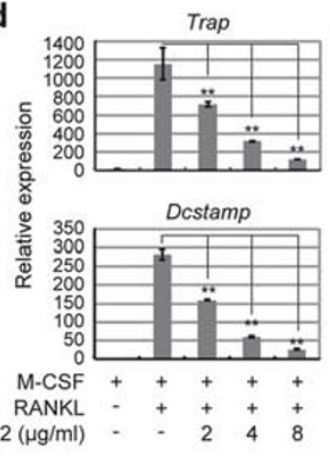

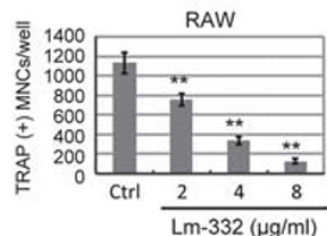
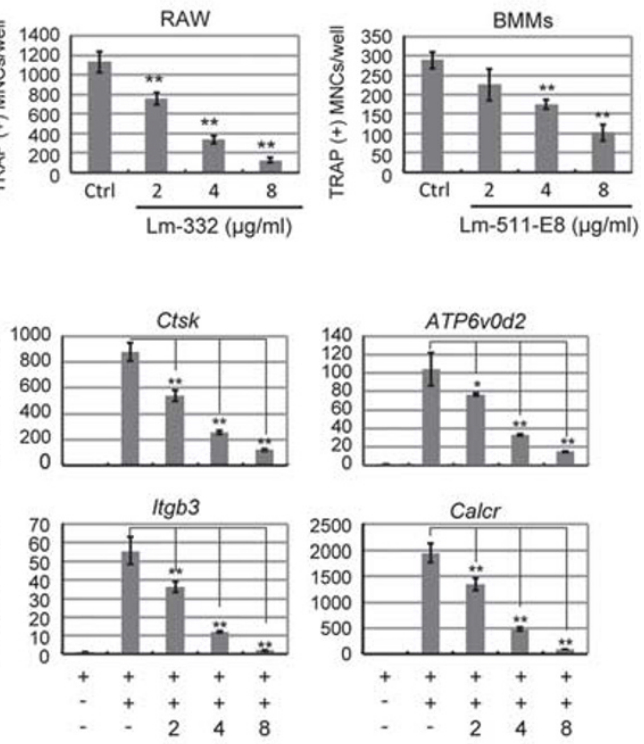

e
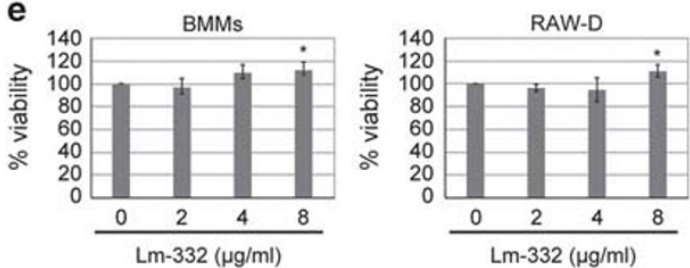

Figure 2 Inhibition of osteoclastogenesis by Laminin-332. BMMs were cultured in 96-well plates coated with indicated concentrations of Lm-332 or Lm-511. BMMs and RAW cells were differentiated into osteoclasts in the presence of $20 \mathrm{ng} / \mathrm{ml} \mathrm{M}$-CSF and $50 \mathrm{ng} / \mathrm{ml} \mathrm{RANKL} \mathrm{for} 4-5 \mathrm{days}$ or $50 \mathrm{ng} / \mathrm{ml}$ RANKL for 3 days. The cells were fixed and stained for TRAP (a), and TRAP-positive multinuclear cells with three or more nuclei were counted (b). The cells were stained for F-actin using Alexa fluor 647-conjugated phalloidin (red) to visualize actin rings and DAPI (blue) for nuclei. The number of MNCs exhibiting actin rings was quantified (c); bars, \pm s.d. ${ }^{*} P<0.01$ by ANOVA with Tukey's test. mRNA expression of osteoclast differentiation-related molecules was analyzed by quantitative real-time PCR. For each target gene, the expression was normalized to $\beta$-2-microglobulin (B2m), and the expression level is shown relative to the control (designated as 1 ) for comparison (d); bars, \pm s.d. ${ }^{* *} P<0.01$ by ANOVA with Tukey's test. BMMs and RAW-D cells $\left(5 \times 10^{3}\right.$ cells/well) were cultured in 96 -well plates coated with indicated concentrations of Lm-332. Viable cells were identified by PrestoBlue fluorescent dye as described in the 'Materials and Methods' section (e); bars, \pm s.d. ${ }^{*} P<0.05$ by ANOVA with Tukey's test.

cultured in the presence of $\beta$-glycerophosphate and ascorbic acid for 14 days to form mature osteoblasts, which secrete calcified matrices. PCR analysis of mature osteoblasts confirmed the expression of osteoblast markers including Runt-related transcription factor 2 (Runx2) and osteocalcin (Bglap). All three Lm-332 chain genes (laminin $\alpha 3$; Lama3, laminin $\beta 3$; Lamb3, laminin $\gamma 2$; Lamc2; Figure 1b). Laminin $\beta 3$ chain was detected in the cytoplasm of mature (ALPpositive) osteoblasts by immunofluorescence (Figure 1c). These data indicate that Lm-332 is likely produced by osteoblasts in bone tissues.

\section{Lm-332 Blocks Osteoclastogenesis in The Presence of RANKL}

To evaluate the role of Lm-332 produced by osteoblasts in bone, we examined the effect of Lm-332 on osteoclastogenesis. Primary BMMs and osteoclast precursor cell line RAW-D cells were cultured on plastic cell culture plates coated with various concentrations $(2-8 \mu \mathrm{g} / \mathrm{ml})$ of $\mathrm{Lm}-332$ in the presence of RANKL. Tartrate-resistant acid phosphatase (TRAP)positive multinucleated cells (MNCs) were generated from BMMs and RAW-D cells in response to RANKL, whereas Lm-332 significantly inhibited the formation of TRAPpositive MNCs in a dose-dependent manner (Figure 2a and b). In addition, osteoclastogenesis was inhibited by Lm-511E8, a truncated laminin composed of the C-terminal regions of the $\alpha, \beta$, and $\gamma$ chains of Lm-511, and having the same affinity for integrin $\alpha 6 \beta 1$ as Lm-332 (Figure 2a and b). We next examined the effect of Lm-332 on actin cytoskeleton organization. MNCs having typical actin rings were observed in control BMMs in response to RANKL; however, Lm-332 suppressed the formation of MNCs with actin rings (Figure 2c). To further examine the inhibitory effect of Lm332 on osteoclastogenesis, we investigated the expression of osteoclast marker genes, TRAP, Cathepsin K (Ctsk), ATPase, $\mathrm{H}+$ transporting, lysosomal V0 subunit D2 (ATP6v0d2), 

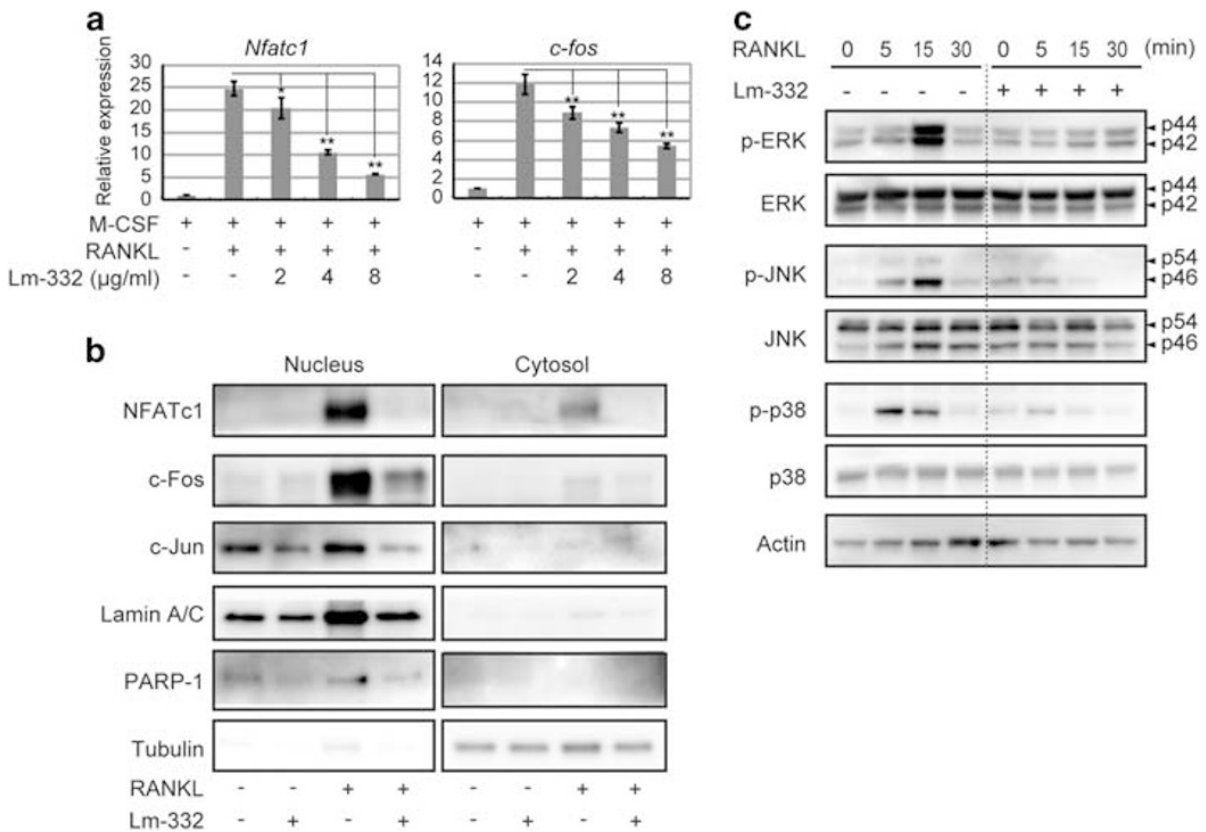

Figure $3 \mathrm{Lm}-332$ suppresses induction and nuclear localization of AP-1 and NFATc1 and blocks activation of MAPKs. (a) BMMs were cultured for osteoclastogenesis $(24 \mathrm{~h}$ ) in 96-well plates coated with the indicated concentrations of Lm-332. mRNA expression of Nfatc1 and c-fos was analyzed by quantitative real-time PCR. For each target gene, the expression was normalized to $B 2 m$, and the expression level is shown relative to the control (designated as 1 ) for comparison; bars, \pm s.d. ${ }^{*} P<0.05 .{ }^{*} P<0.01$ by ANOVA with Tukey's test. (b) BMMs were cultured for $24 \mathrm{~h}$ with M-CSF and RANKL in 96-well plates coated with $\mathrm{Lm}-332(8 \mu \mathrm{g} / \mathrm{ml})$ as indicated. Nuclear and cytosolic fractions were extracted and translocation of NFATc1, C-Fos, and c-Jun were analyzed by western blotting. Purity of nuclear or cytosol fractions were evaluated by analyzing Lamin A/C, PARP-1, and Tubulin. (c) BMMs were starved in a-MEM supplemented with $0.1 \% \mathrm{FBS}$ for $5 \mathrm{~h}$ in $\mathrm{Lm}$-332-coated $(8 \mu \mathrm{g} / \mathrm{ml}$ ) six-well plates, and stimulated with RANKL (300 $\mathrm{ng} / \mathrm{ml})$ for indicated times. Lysates were prepared and subjected to western blot analysis with the indicated antibodies. Actin was used as an internal control.

Dendritic cell-specific transmembrane protein (Dcstamp), Integrin $\beta 3$ (Itgb3), and Calcitonin receptor (Calcr). RANKL induced expression of osteoclast marker gene mRNA in BMMs cultured on Lm-332-non-coated culture plates (noncoated plates); whereas, coating plates with Lm-332 significantly suppressed the capacity of RANKL to induce expression of these genes (Figure 2d). Lm-332 did not exhibit any cytotoxicity against BMMs and RAW-D cells, but rather slightly promoted proliferation of both BMMs and RAW-D cells (Figure 2e), indicating that Lm-332 markedly suppressed osteoclastogenesis without diminishing cell viability.

\section{Lm-332 Suppresses Induction and Nuclear Localization of AP-1 and NFATc1 in Response to RANKL}

We next examined whether Lm-332 affected the expression in osteoclasts of two fundamental transcription factors downstream of RANKL signaling, AP-1 and NFATc1. Marked increases in $N f a t c 1$ and $c$-fos mRNA levels were observed in BMMs cultured on non-coated plates 1 day after stimulation with RANKL; whereas, on Lm-332-coated plates their induction was suppressed in a dose-dependent manner (Figure 3a). Induction and nuclear localization of NFATc1, c-Fos, and c-Jun proteins in response to RANKL was potently blocked by Lm-332 in BMMs (Figure 3b).

\section{Lm-332 Blocks RANKL-Induced Activation of MAPK Pathways}

RANKL stimulation triggers formation of a RANK-TRAF6 complex, which results in the activation of various downstream molecules, including MAPKs. ${ }^{31}$ We next investigated whether Lm-332 affected MAPK signaling pathways in response to RANKL. BMMs were starved and then stimulated with RANKL. RANKL-induced phosphorylation of ERK, JNK, and p38MAPK was observed within 5 to $15 \mathrm{~min}$ in BMMs cultured on non-coated plates; whereas, the RANKLinduced phosphorylation of these MAPKs was markedly suppressed on Lm-332-coated plates (Figure 3c). These data suggested that Lm-332 blocked early signaling events induced by RANKL, such as activation of MAPKs.

\section{Lm-332 Attenuates Rank Expression and Retains a Macrophage Phenotype Even in the Presence of RANKL} Adhesion signaling mediated by $\alpha_{\mathrm{v}} \beta_{3}$ integrin upregulates Rank expression, which is essential for RANKL-induced osteoclast differentiation. ${ }^{32,33}$ Laminins are also important transducers of adhesion signals through their receptors, including integrins, in many types of cells. We speculated that Lm-332 might affect Rank mRNA expression in osteoclast precursors. To determine this, the expression levels of Rank mRNA in BMMs and RAW-D cells were analyzed. We found that BMMs and RAW-D cells cultured on 
non-coated plates exhibited a marked increase in Rank mRNA levels in response to RANKL, whereas Rank mRNA expression was significantly suppressed on Lm-332-coated plates in a dose-dependent manner (Figure 4a). When BMMs were stimulated with RANKL under the non-coated condition, the expression level of Rank mRNA peaked at 2 days and remained high through day 4 (Figure 4b). However, BMMs cultured on Lm-332-coated plates failed to induce Rank mRNA in response to RANKL (Figure $4 \mathrm{~b}$ ).

To determine whether Lm-332 affected commitment to osteoclast differentiation in response to RANKL, we examined the macrophage markers nonspecific esterase (NSE) activity and expression of lysozyme (Lyz) and Emrl (Figure 5). When BMMs were cultured on non-coated plates, the proportion of NSE-positive cells was $\sim 80 \%$ before RANKL stimulation (day 0 ); the proportion of NSE-positive cells had significantly decreased (to $\sim 20 \%$ ) by day 4 after treatment with RANKL, and was accompanied by formation of osteoclasts (Figure $5 \mathrm{a}$, left panel). In contrast, BMMs cultured on Lm-332-coated plates retained NSE activity at day 4 as compared with BMMs before RANKL stimulation (day 0). BMMs cultured on noncoated plates stimulated with RANKL formed osteoclasts and exhibited decreased expression of macrophage marker genes, such as Lyz and Emr1. In contrast, on day 4, the expression of
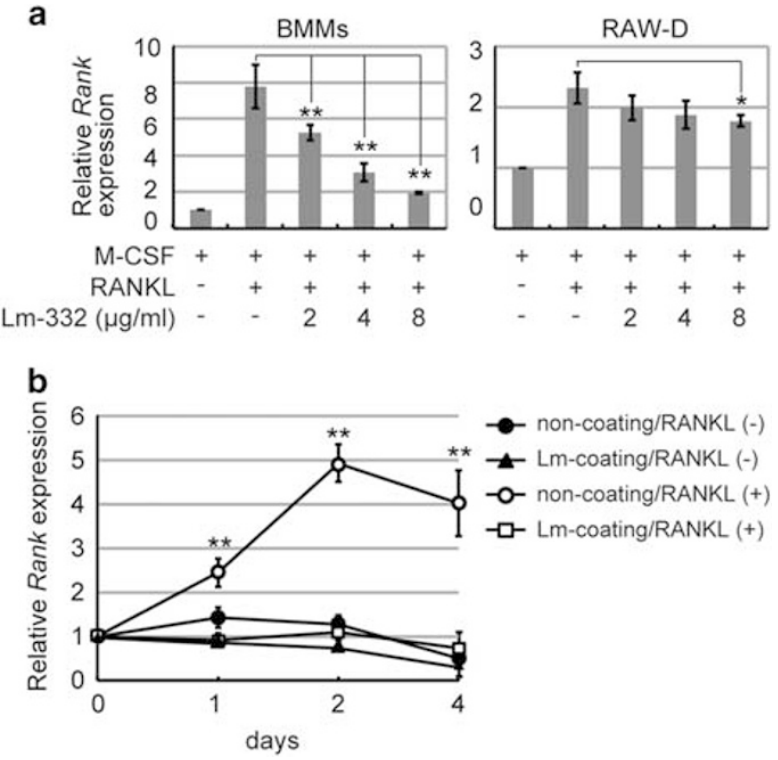

Figure 4 Lm-332 attenuates RANK expression after RANKL stimulation. BMMs and RAW cells were cultured in plates coated with the indicated concentrations of $\mathrm{Lm}-332$ in the presence of M-CSF $(20 \mathrm{ng} / \mathrm{ml})$ and RANKL $(50 \mathrm{ng} / \mathrm{ml}$ ) for 4 days. (a) mRNA expression of Rank was analyzed by quantitative real-time PCR. The expression was normalized to $B 2 m$, and the expression level is shown relative to the control (designated as 1) for comparison; bars, \pm s.d. ${ }^{*} P<0.05 .{ }^{*} P<0.01$ by ANOVA with Tukey's test. (b) At each time point, mRNA expression of Rank was analyzed. Expression was normalized to $B 2 m$, and the expression level is shown relative to the day 0 level (designated as 1 ) for comparison. ${ }^{* *} P<0.01$ compared to BMMs cultured in Lm-332-coated plates in the presence of RANKL by ANOVA with Tukey's test.
Lyz and Emrl was not affected when BMMs were cultured on Lm-332-coated plates even in the presence of RANKL (Figure $5 \mathrm{~b}$ ). These results suggested that Lm-332 selectively blocked osteoclastogenesis without affecting macrophage differentiation from bipotential precursors.

\section{Osteoclastogenic Factors Suppress Lm-332 Expression in Osteoblasts}

RANKL expression is known to be induced by bone resorbing factors, such as $1 \alpha, 25$-dihydroxyvitamin $\mathrm{D}_{3}\left(1,25(\mathrm{OH})_{2} \mathrm{D}_{3}\right)$ or parathyroid hormone (PTH) ${ }^{34,35}$ In light of the inhibitory effect of Lm-332 on osteoclastogenesis and the expression of Lm-332 in osteoblasts, we analyzed expression of three components of Lm-332 in osteoblasts after treatment with $1,25(\mathrm{OH})_{2} \mathrm{D}_{3}$ or PTH. Expression of all three laminin-332 chains (Lama3, Lamb3, and Lamc2) was markedly suppressed after treatment of osteoblasts with $1,25(\mathrm{OH})_{2} \mathrm{D}_{3}$ (Figure 6a). Similarly, the expression of all three laminin-332 chains (Lama3, Lamb3, and Lamc2) was also suppressed by PTH treatment (Figure 6b). These data indicated that the osteoclastogenic factors that act by inducing RANKL expression efficiently suppressed production of Lm-332, a key negative regulator of osteoclast differentiation.

\section{DISCUSSION}

We provide evidence that laminin $\beta 3$, a specific chain of Lm332 , is present in osteoblast-like cells localized on the bone surface. In our in vitro studies, we confirmed that Lm-332 was expressed in primary osteoblasts. We also obtained lines of evidence indicating that $\mathrm{Lm}-332$ had the ability to suppress osteoclastogenesis. In normal osteoclastogenesis, osteoclasts are formed on bone surface. Lm-332 produced from osteoblasts is thought to contribute to a fine modulation of osteoclastogenesis in normal bone remodeling.

We have clarified that both Lm-332 and Lm-511-E8 exert an inhibitory effect on osteoclastogenesis. Among the subunits of laminin, the C-terminal of laminin $\alpha$ chain is utilized for binding to integrins. ${ }^{5} \mathrm{Lm}-332$ preferentially binds to integrin $\alpha 3 \beta 1$ and/or $\alpha 6 \beta 1$, and proteolytically processed $\alpha 3$ laminin G (LG) domains bind to integrin $\alpha 6 \beta 4 .{ }^{36} \mathrm{Lm}-511$ $\mathrm{E} 8$ is a truncated protein composed of the $\mathrm{C}$-terminal regions of the $\alpha, \beta$, and $\gamma$ chains of Lm-511 that retain the full capability and high affinity for binding to integrin $\alpha 6 \beta 1 .^{37,38}$ Suppression of osteoclastogenesis induced by Lm-332 or $\mathrm{Lm}-511$ is thought to be mediated through integrin $\alpha 6 \beta 1$. We have confirmed the expression of integrin $\alpha 6$ and $\beta 1$ in BMMs (data not shown).

Lm-332 blocked RANKL-induced phosphorylation of MAPKs (ERK, JNK, and p38) in BMMs as the early event. Lm-332 suppressed RANKL-induced expression of NFATc1 and $c-f o s$ and blocked nuclear localization of these transcription factors. These events are considered to be the main mechanism for inhibition of osteoclastogenesis. It will be of interest to elucidate the types of intracellular signaling that follow binding of Lm-332 to the receptor expressed on 
a

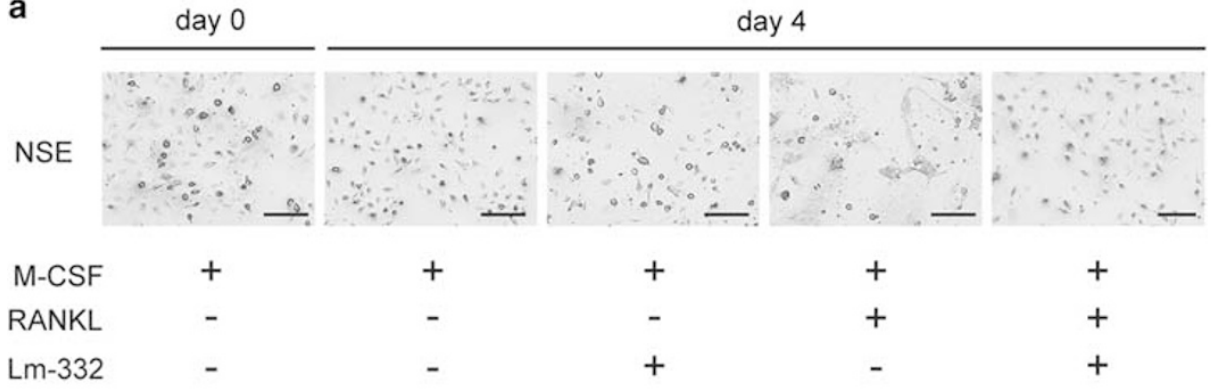

b

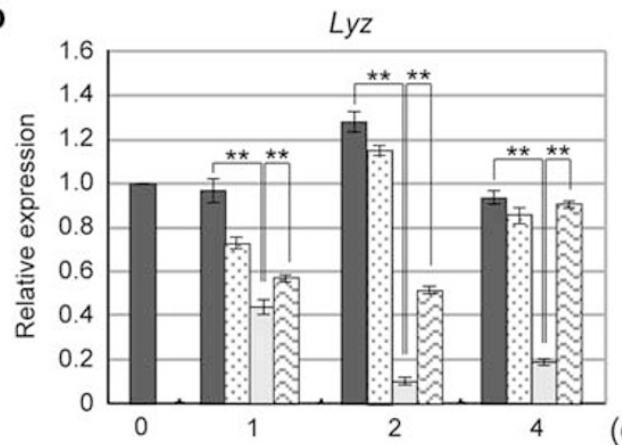

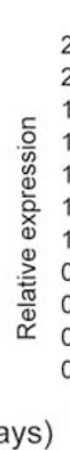

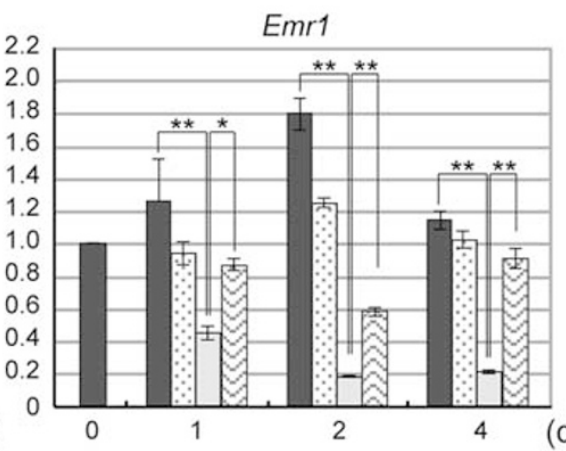

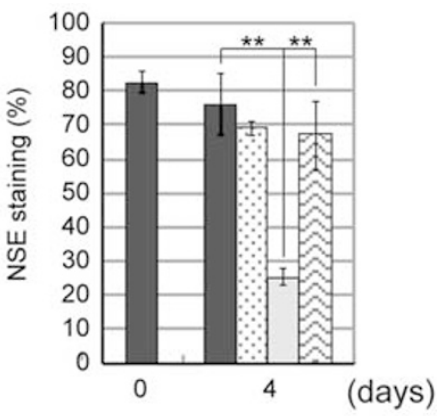

$\square$ non-coated/RANKL (-)

Lm-coated/RANKL (-)

non-coated/RANKL (+)

Lm-coated/RANKL (+)

Figure 5 BMMs cultured in Lm-332-coated plates retain macrophage characteristics in the presence of RANKL. (a) Nonspecific esterase activity was determined using the $a$-Naphthyl Acetate Esterase Kit (Sigma) according to the manufacturer's instructions. BMMs exhibit activity of the macrophagespecific enzyme $a$-NSE, which is detectable by characteristic black cytoplasmic staining (left); bars, $50 \mu \mathrm{m}$. The proportion of NSE-positive cells to total cells at day 4 was quantified (right); bars, \pm s.d. ${ }^{*} P<0.01$ by ANOVA with Tukey's test. (b) BMMs were cultured in the presence of M-CSF ( $\left.20 \mathrm{ng} / \mathrm{ml}\right)$ and RANKL $(50 \mathrm{ng} / \mathrm{ml})$ in $\mathrm{Lm}$-332-coated $(2-8 \mu \mathrm{g} / \mathrm{ml})$ or non-coated plates for 1, 2, or 4 days. mRNA expression of macrophage markers (Lyz and Emr1) was analyzed by quantitative real-time PCR. For each target gene, the expression was normalized to $B 2 m$, and the expression level is shown relative to the control (designated as 1 ) for comparison; bars, \pm s.d. ${ }^{* *} P<0.01$ by ANOVA with Tukey's test.

osteoclast precursors. Lm-332 also blocked the induction of RANK in BMMs stimulated with RANKL, suggesting that an adhesion signal through Lm-332 might affect RANK induction. Mochizuki et al. reported that a cell adhesion signal, possibly through $\alpha \mathrm{v} \beta 3$ integrin, is essential for RANK induction in osteoclast precursors during RANKL-induced osteoclastogenesis. ${ }^{33}$ Interestingly, they showed that BMMs cultured under non-adherent conditions retained mature macrophage phenotypes and failed to express Rank mRNA. Similar to their report, we found that BMMs cultured on Lm332-coated plates failed to induce Rank mRNA even under RANKL-stimulation, and the BMMS retained macrophage phenotypes evidenced by NSE activity and expression of lyz and Emr1. Our results show that Lm-332 maintains macrophage phenotypes of BMMs even in the presence of RANKL. Under the non-coated culture condition, BMMs exhibited significant induction of Rank mRNA ( 8 -fold) in response to RANKL-stimulation, whereas in RAW-D cells the induction was moderate ( $\sim 2$-fold). This might be due to differences in the cell population between BMMs and RAWD. BMMs are M-CSF responsive cells prepared from bone marrow cells, in which the number of RANK-positive osteoclast precursors is low before exposure to RANKL. When these cells were treated with RANKL in the presence of
M-CSF stimulation, Rank-expressing early osteoclast precursors proliferated to form large numbers of RANK-positive late precursors. In contrast, RAW-D is an osteoclast precursor cell line that was established from one cell that was highly responsive to RANKL-stimuli. As RAW-D cells at baseline express high levels of Rank mRNA, ${ }^{28}$ it is reasonable that the Rank mRNA level upregulated only twofold in response to RANKL. It will be important to determine whether upregulaton of Rank mRNA expression by RANKL is suppressed by Lm-332 by use of the reporter assay system using Rank promoter.

It has been proposed that there are two types of bone marrow niches for HSC maintenance: the endosteal niche, populated by osteoblasts, and the vascular niche, which includes sinusoids and endothelial cells. ${ }^{1}$ Osteoclasts are derived from HSCs, and their differentiation is supported by bone marrow stromal cells or osteoblasts through cell-cell interactions. ${ }^{18}$ In the current study, immunohistochemical analysis for laminin $\beta 3$ indicated that the fluorescence signal was detected in cells lining limited areas of the bone surface, indicating that Lm-332 is possibly expressed in a subset of cells in the osteoblast lineage. We have confirmed the expression of all chains of Lm-332 in primary osteoblasts by use of RT-PCR and immunofluorescence staining. 

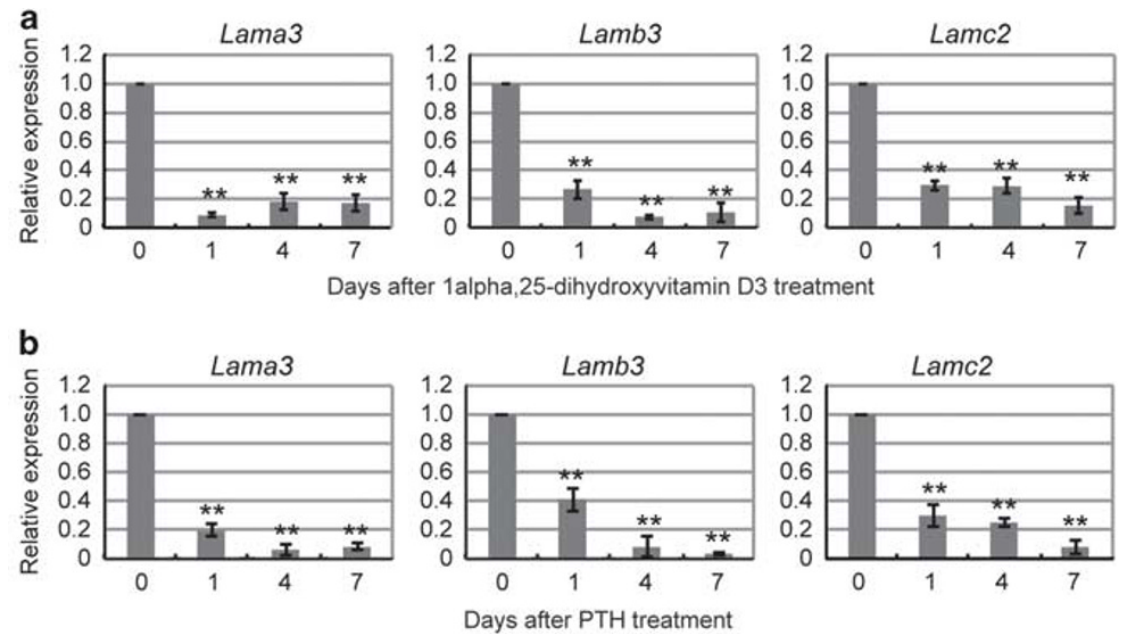

C
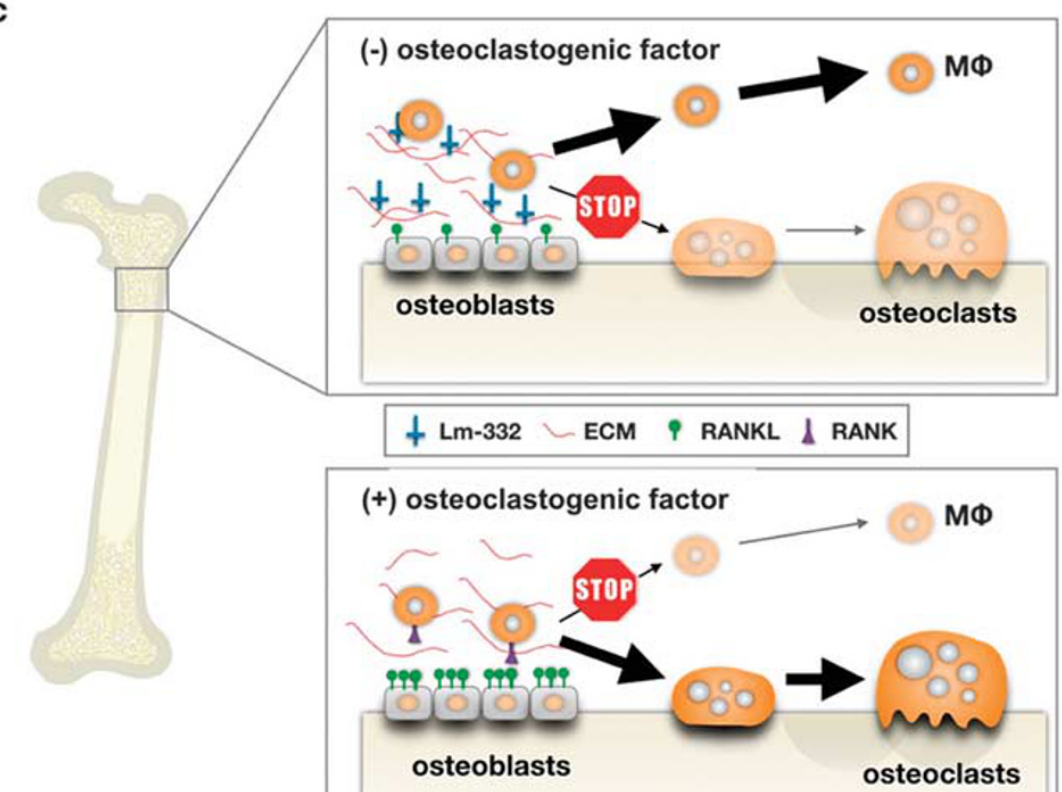

Figure 6 Suppression of Lm-332 expression by treatment with osteoclastogenic factors. Osteoblasts prepared from calvaria of newborn mice were treated with $10 \mathrm{nM}$ of $1 a, 25$-dihydroxyvitamin $\mathrm{D}_{3}$ (a) or $100 \mathrm{ng} / \mathrm{ml}$ of PTH (b) for 7 days. mRNA expression of laminin- $\alpha 3$ (Lama3), - $\beta 3$ (Lamb3), and - $\gamma 2$ (Lamc2) was analyzed by quantitative real-time PCR. For each target gene, the expression was normalized to $A c t b$, and the expression level is shown relative to the control of day 0 culture (designated as 1 ) for comparison; bars, \pm s.d. ${ }^{* * P} P 0.01$ by paired $t$-test. (c) Hypothetical model of the role of $L m$ - 332 in modulation of osteoclastogenesis. In the absence of osteoclastogenic factors, $\mathrm{Lm}-332$ secreted by osteoblasts suppresses osteoclastogenesis and supports the maintenance of the macrophage population. In the presence of osteoclastogenic factors such as 1a,25-dihydroxyvitamin $\mathrm{D}_{3}$ or PTH, Lm-332 production in osteoblasts is markedly suppressed, which allows promotion of osteoclastogenesis through RANK/RANKL signaling. $M \Phi$, macrophage.

Osteoblasts secrete proteins such as OPG and Semaphorin 3A, both of which exert osteoprotective effects by suppressing osteoclast differentiation and function. ${ }^{39}$ Osteoclast differentiation is induced by RANKL, which is expressed by osteoblasts and bone marrow stromal cells in response to osteotropic factors such as $1,25(\mathrm{OH})_{2} \mathrm{D}_{3}$, prostagrandin $\mathrm{E} 2$ or $\mathrm{PTH}$. All three components of Lm-332 were detected in primary osteoblasts, but their expression was markedly suppressed by treatment with osteoclastogenic factors that induce RANKL, such as $1,25(\mathrm{OH})_{2} \mathrm{D}_{3}$ and PTH. As illustrated in our model shown in Figure 6c, the interplay of these factors determines the formation or inhibition of osteoclasts (Figure 6c). Our findings suggest that Lm-332 is a novel osteoprotective factor produced by osteoblasts.

The results presented here provide novel evidence indicating that Lm-332 exerts inhibitory effects on RANKL-induced osteoclastogenesis. This effect was accompanied by inhibition of induction of Rank mRNA expression and retention of 
macrophage phenotypes by BMMs. In addition, Lm-332 was expressed by primary osteoblasts in culture, and its expression was negatively regulated by osteoclastogenic factors, suggesting that Lm-332 is a novel negative regulator that may define osteoclastogenesis spatiotemporally in bone tissues. It will be important to elucidate the molecular mechanisms by which adhesion signaling via Lm-332 regulates osteoclastogenesis. Insights from our findings may contribute to the development of strategies for the fine regulation of bone metabolism.

\section{ACKNOWLEDGMENTS}

This work was supported in part by a Grant-in-Aid for Scientific Research (C) (24591923 and 15K11014; to NU). This work was also supported in part by a Grant-in-Aid for Challenging Exploratory Research (15K15677).

\section{DISCLOSURE/CONFLICT OF INTEREST}

The authors declare no conflict of interest.

1. Wang LD, Wagers AJ. Dynamic niches in the origination and differentiation of haematopoietic stem cells. Nat Rev Mol Cell Biol 2011;12:643-655.

2. Klamer $S$, Voermans $C$. The role of novel and known extracellular matrix and adhesion molecules in the homeostatic and regenerative bone marrow microenvironment. Cell Adh Migr 2014;8:563-577.

3. Prosper $\mathrm{F}$, Verfaillie $\mathrm{CM}$. Regulation of hematopoiesis through adhesion receptors. J Leukoc Biol 2001;69:307-316.

4. Tryggvason K. The laminin family. Curr Opin Cell Biol 1993;5:877-882.

5. Aumailley M. The laminin family. Cell Adh Migr 2013;7:48-55.

6. Shaw LM, Messier JM, Mercurio AM. The activation dependent adhesion of macrophages to laminin involves cytoskeletal anchoring and phosphorylation of the alpha 6 beta 1 integrin. J Cell Biol 1990;110:2167-2174.

7. Siler U, Seiffert M, Puch S, et al. Characterization and functional analysis of laminin isoforms in human bone marrow. Blood 2000;96:4194-4203.

8. Gu YC, Kortesmaa J, Tryggvason K, et al. Laminin isoform-specific promotion of adhesion and migration of human bone marrow progenitor cells. Blood 2003;101:877-885.

9. Mizushima H, Koshikawa N, Moriyama K, et al. Wide distribution of laminin-5 gamma 2 chain in basement membranes of various human tissues. Horm Res 1998;50(Suppl 2):7-14.

10. Morgner J, Ghatak S, Jakobi T, et al. Integrin-linked kinase regulates the niche of quiescent epidermal stem cells. Nat Commun 2015;6:8198.

11. Govaere O, Wouters J, Petz M, et al. Laminin-332 sustains chemoresistance and quiescence as part of the human hepatic cancer stem cell niche. J Hepatol 2016;64:609-617.

12. Spessotto $P$, Zucchetto $A$, Degan $M$, et al. Laminin-332 (Laminin-5) is the major motility ligand for B cell chronic lymphocytic leukemia. Matrix Biol 2007;26:473-484.

13. Klees RF, Salasznyk RM, Kingsley K, et al. Laminin-5 induces osteogenic gene expression in human mesenchymal stem cells through an ERKdependent pathway. Mol Biol Cell 2005;16:881-890.

14. Siler $\mathrm{U}$, Rousselle $\mathrm{P}$, Muller $\mathrm{CA}$, et al. Laminin gamma2 chain as a stromal cell marker of the human bone marrow microenvironment. $\mathrm{Br}$ J Haematol 2002;119:212-220.

15. Asagiri M, Takayanagi $\mathrm{H}$. The molecular understanding of osteoclast differentiation. Bone 2007;40:251-264.

16. Yasuda H, Shima N, Nakagawa N, et al. Osteoclast differentiation factor is a ligand for osteoprotegerin/osteoclastogenesis-inhibitory factor and is identical to TRANCE/RANKL. Proc Natl Acad Sci USA 1998;95:3597-3602.

17. Yoshida $H$, Hayashi $S$, Kunisada $T$, et al. The murine mutation osteopetrosis is in the coding region of the macrophage colony stimulating factor gene. Nature 1990;345:442-444.
18. Suda T, Takahashi N, Udagawa N, et al. Modulation of osteoclast differentiation and function by the new members of the tumor necrosis factor receptor and ligand families. Endocr Rev 1999;20: 345-357.

19. Grigoriadis $A E$, Wang ZQ, Cecchini MG, et al. c-Fos: a key regulator of osteoclast-macrophage lineage determination and bone remodeling. Science 1994;266:443-448.

20. Ikeda F, Nishimura R, Matsubara T, et al. Critical roles of c-Jun signaling in regulation of NFAT family and RANKL-regulated osteoclast differentiation. J Clin Invest 2004;114:475-484.

21. Takayanagi $\mathrm{H}, \mathrm{Kim} \mathrm{S}$, Koga $\mathrm{T}$, et al. Induction and activation of the transcription factor NFATc1 (NFAT2) integrate RANKL signaling in terminal differentiation of osteoclasts. Dev Cell 2002;3: 889-901.

22. Rodan SB, Rodan GA. Integrin function in osteoclasts. J Endocrinol 1997;154 Suppl:S47-S56.

23. Butler WT. The nature and significance of osteopontin. Connect Tissue Res 1989;23:123-136.

24. Oldberg A, Franzen A, Heinegard D. The primary structure of a cell-binding bone sialoprotein. J Biol Chem 1988;263:19430-19432.

25. Kukita T, Hata K, Kukita A, et al. Laminin, a major basement membrane component of the blood vessel, as a negative regulator of osteoclastogenesis. Calcif Tissue Int 1998;63:140-142.

26. Uehara N, Matsuoka Y, Tsubura A. Mesothelin promotes anchorageindependent growth and prevents anoikis via extracellular signalregulated kinase signaling pathway in human breast cancer cells. Mol Cancer Res 2008;6:186-193.

27. Kukita T, Wada N, Kukita A, et al. RANKL-induced DC-STAMP is essential for osteoclastogenesis. J Exp Med 2004;200:941-946.

28. Watanabe $T$, Kukita $T$, Kukita $A$, et al. Direct stimulation of osteoclastogenesis by MIP-1alpha: evidence obtained from studies using RAW264 cell clone highly responsive to RANKL. J Endocrinol 2004;180:193-201.

29. Uehara N, Unami A, Kiyozuka Y, et al. Parous mammary glands exhibit distinct alterations in gene expression and proliferation responsiveness to carcinogenic stimuli in Lewis rats. Oncol Rep 2006;15:903-911.

30. Uehara N, Kanematsu S, Miki H, et al. Requirement of p38 MAPK for a cell-death pathway triggered by vorinostat in MDA-MB-231 human breast cancer cells. Cancer Lett 2012;315:112-121.

31. Takayanagi $\mathrm{H}$. The role of NFAT in osteoclast formation. Ann N Y Acad Sci 2007;1116:227-237.

32. Miyamoto T, Arai $\mathrm{F}$, Ohneda $\mathrm{O}$, et al. An adherent condition is required for formation of multinuclear osteoclasts in the presence of macrophage colony-stimulating factor and receptor activator of nuclear factor kappa B ligand. Blood 2000;96:4335-4343.

33. Mochizuki $A$, Takami M, Miyamoto $Y$, et al. Cell adhesion signaling regulates RANK expression in osteoclast precursors. PLoS ONE 2012;7: e48795.

34. Takahashi $\mathrm{N}$, Akatsu $\mathrm{T}$, Udagawa $\mathrm{N}$, et al. Osteoblastic cells are involved in osteoclast formation. Endocrinology 1988;123:2600-2602.

35. Udagawa $\mathrm{N}$, Takahashi $\mathrm{N}$, Akatsu $\mathrm{T}$, et al. The bone marrow-derived stromal cell lines MC3T3-G2/PA6 and ST2 support osteoclast-like cell differentiation in cocultures with mouse spleen cells. Endocrinology 1989;125:1805-1813.

36. Hintermann E, Quaranta V. Epithelial cell motility on laminin-5: regulation by matrix assembly, proteolysis, integrins and erbB receptors. Matrix Biol 2004;23:75-85.

37. Taniguchi $Y$, Ido $\mathrm{H}$, Sanzen $\mathrm{N}$, et al. The $\mathrm{C}$-terminal region of laminin beta chains modulates the integrin binding affinities of laminins. J Biol Chem 2009;284:7820-7831.

38. Nishiuchi R, Takagi J, Hayashi $M$, et al. Ligand-binding specificities of laminin-binding integrins: a comprehensive survey of laminin-integrin interactions using recombinant alpha3beta1, alpha6beta1, alpha7beta 1 and alpha6beta4 integrins. Matrix Biol 2006;25:189-197.

39. Hayashi M, Nakashima $T$, Taniguchi $M$, et al. Osteoprotection by semaphorin 3A. Nature 2012;485:69-74. 